\title{
Redefiniendo el catálogo. Expectativas de las interfaces de descubrimiento centradas en el usuario
}

\author{
Ma Luisa Alvite Díez *
}

Artículo recibido:

7 de marzo de 2012.

Artículo aceptado:

16 de abril de 2012.

\section{RESUMEN}

Los continuos desarrollos de los catálogos en línea (OPAC) no han logrado aún conformar una herramienta de búsqueda óptima para el conjunto de los recursos disponibles en una biblioteca universitaria del siglo XXI. La comunidad académica percibe el catálogo como un instrumento ineficaz y alejado de los servicios web que emplea cotidianamente. Se repasan las tentativas de desarrollo de los OPAC y la incorporación de metabuscadores en las sedes web de las bibliotecas académicas. Se indaga en la creciente implementación de interfaces de descubrimiento, revisando los estudios realizados sobre este tipo de plataformas, funcionalidades e implementación, de un lado, y análisis de usabilidad, del otro. Se examina finalmente el

* Universidad de León, España. luisa.alvite@unileon.es

INVESTIGACIÓN BIBLIOTECOLÓGICA, Vol. 26, Núm. 56, enero/abril, 2012, México, ISSN: 0187-358X. pp. 181-204 
estado actual de las herramientas de búsqueda en las bibliotecas universitarias españolas coligiendo de la exploración la tendencia clara hacia la instalación de herramientas de búsqueda única.

Palabras clave: Bibliotecas universitarias; Catálogos en línea; Interfaces de descubrimiento; Metabuscadores; Sistemas Integrados de Gestión Bibliotecaria.

\section{ABSTRACT \\ Redefining the Catalog. Expectations on User-Cen- tered Discovery Interfaces $M^{a}$ Luisa Alvite Diez}

The continuous developments of OPACs still have not succeeded to constitute an optimal search tool for the overall available resources on a twenty-first century academic library. The academic community perceives OPAC as an ineffective tool and far from the web services that they usually employ. The attempts to develop OPACs and incorporation of metasearch products on the websites of academic libraries are reviewed. The paper explores the increasing implementation of discovery interfaces, reviewing studies on these platforms, its functionality and implementation on one side, and usability analysis on the other. The current state of search tools in the Spanish academic libraries is finally examined, deducing of this exploration the clear trend towards the implementation of single search tools.

Keywords: Academic Libraries; Online Catalogs; Discovery Interfaces; Federated Search Systems; Integrated Library System; OPAC.

\section{LA PERCEPCIÓN DEL CATÁLOGO POR PARTE DEL USUARIO} radicionalmente ha sido el catálogo la herramienta de búsqueda prioritaria de la biblioteca. Gracias a Internet y a los desarrollos tecnológicos continuados, la última década ha permitido que los catálogos en línea presenten 
transformaciones radicales, acceso a colecciones ingentes de recursos de información y mejoras indiscutibles en sus interfaces.

Las bibliotecas, específicamente las universitarias, han realizado enormes inversiones para adaptarse a los cambios tecnológicos exigidos por la Web, sin embargo, colecciones académicas y servicios bibliotecarios parecen ocupar un papel marginal en el mercado del consumo de la información electrónica. Siendo notables los avances realizados y reseñables las mejoras tecnológicas implementadas en los OPAC, la realidad revela dificultades en los catálogos en línea, la coincidencia y persistencia en los resultados de las investigaciones en tres décadas de existencia resultan incontestables: el usuario final encuentra difícil el uso de los catálogos, y además el éxito de la Web ha conducido al declive y desplazamiento del OPAC como elemento de primer orden en la recuperación de información.

Se constatan dos tendencias que afectan a la consideración del OPAC como herramienta nuclear de recuperación en la biblioteca: integración y diversificación. El catálogo de las bibliotecas híbridas integra información tradicional y electrónica, física y virtual, en propiedad y bajo licencia; y por otra parte, el usuario puede acceder desde el portal de la biblioteca a bases de datos suscritas por la institución, plataformas de proveedores de contenidos electrónicos, otros catálogos, repositorios y recursos. Esta situación reviste un importante grado de complejidad para el usuario final cuando se trata de comprender la funcionalidad y los valores añadidos del OPAC frente a otras herramientas de recuperación, y de elegir el catálogo en línea como primer elemento de búsqueda, incluso dentro del portal de la biblioteca.

Para Novotny (2004), el interés mostrado por los OPAC comenzó a disminuir cuando la preocupación en las bibliotecas, a finales de la década de los 90 del pasado siglo, se focalizó en la inclusión de recursos electrónicos en las colecciones. En este periodo ha irrumpido una nueva generación de usuarios en los campus universitarios que espera que los catálogos en línea funcionen como un motor de búsqueda.

Borgman (1996) retoma los argumentos expuestos en su estudio de 1986 para confirmar las manifiestas dificultades que se encuentran al emplear los catálogos en línea una década después. Las mejoras en las interfaces, según la profesora Borgman, han sido superficiales y no han atendido a las funcionalidades esenciales del OPAC, cuyos sistemas de búsqueda están diseñados para los bibliotecarios profesionales con un sólido marco conceptual para la recuperación de información. El informe de las Bibliotecas de la Universidad de California (2005, p. 7) es contundente al respecto: los actuales catálogos de bibliotecas están deficientemente diseñados para hacer tareas de búsqueda, recuperación y selección en el creciente conjunto de recursos disponibles en 
nuestras bibliotecas. Se adaptan mejor a la localización y obtención de ítems conocidos y, tanto para bibliotecarios como para usuarios, el catálogo es solamente una opción más de acceso a nuestras colecciones. En el informe de Calhoun (2006), comisionado por la Biblioteca del Congreso de Estados Unidos, se subraya cómo los usuarios acusan al catálogo de contener sobre todo referencias y apenas permitir búsquedas de texto completo. En el mismo trabajo se insiste en que la interfaz del catálogo sea éste único o colectivo “debería parecerse a y trabajar como Google". Los usuarios esperan una satisfacción instantánea y un feedback positivo de los sistemas que emplean, (Calhoun, 2006, p. 38).

Igualmente se han hecho notar los aspectos comunes de los catálogos con el modelo exitoso de librería virtual liderado por Amazon. Zumer (2007) se detiene en seis características de Amazon completamente extrapolables a los OPAC: simplicidad; imagen de las cubiertas de los libros; recomendaciones basadas en el seguimiento del comportamiento de los usuarios; revisionesrecomendaciones elaboradas por los lectores; ordenación de los resultados por popularidad y disponibilidad de los contenidos, y búsqueda en el texto completo.

El debate tiene su correlato en el ámbito de la descripción bibliográfica y así en las conclusiones del informe de Calhoun et al (2009, p. 51), se subrayan diferencias significativas entre las prioridades cualitativas establecidas por usuarios y bibliotecarios en la utilización del catálogo, así como entre los datos que éste debe proporcionar. Flynn (2010) apela a la necesidad urgente de repensar el modelo de catalogación, y de abandonar los catálogos locales en favor de catálogos unificados con valores añadidos y mayor visibilidad en el entorno electrónico, y que eviten, además, la duplicación de esfuerzos. En este sentido, el estudio del Research Information Network (2009, p. 37) señala con contundencia que los procesos actuales de creación de registros bibliográficos son imperfectos e ineficaces, y que inciden en la disminución del valor y la utilidad para el usuario final de los catálogos de bibliotecas individuales, y aboga por una redefinición de los estándares y la calidad de los registros que nos permitan plantear la disponibilidad de tener catálogos compartidos. El trabajo de RIN insiste en la necesidad de desarrollar modelos de negocio en los que colaboren bibliotecas, editores, agregadores y distribuidores de contenidos o empresas como Google, entre otros.

Parece que en cierto modo la perspectiva de los bibliotecarios se mantiene demasiado aferrada a los principios clásicos de organización de la información, mientras que las expectativas de los usuarios están claramente influenciadas por las herramientas y servicios web que emplean. Campbell y Fast (2004) se refieren en este sentido a una muy limitada adaptación de 
los catálogos al entorno web, OPAC codificados en formato MARC y con un diseño enraizado en la tradicional teoría y práctica de la recuperación de información.

\section{SOLUCIONES APORTADAS POR LOS SISTEMAS DE GESTIÓN BIBLIOTECARIA}

Rowley (1998) sitúa la época de transformación de los sistemas integrados de gestión bibliotecaria (SIGB) a mediados de la década de los años noventa, momento de transición de los sistemas de tercera generación a los de cuarta generación, en paralelo a la expansión de la Web y a la tendencia imparable hacia la estandarización e interoperabilidad. Los SIGB en el momento actual se asientan en la arquitectura cliente/servidor, el uso de sistemas operativos no propietarios que posibilitan la interoperabilidad y la integración de distintas plataformas en un mismo sistema.

A los módulos tradicionales, los vendedores les han venido sumando un conjunto de aplicaciones que les permiten a las bibliotecas despegarse de interfaces desfasadas sin reemplazar el conjunto del sistema. En esta línea, el trabajo de Garza (2009) documenta la exitosa implementación de Drupal ${ }^{1}$ y otras interfaces de programación de aplicaciones (API) públicas en una biblioteca académica mostrando la integración con Millenium WebOpac, OCLC, LibraryThing, herramientas de Google, etcétera.

Creemos que han sido dos los principales retos de los SIGB en los últimos años: gestionar adecuadamente colecciones electrónicas y mejorar las interfaces de los OPAC. Salse Rovira (2005), señala cómo son precisamente los módulos del OPAC los que presentan más variaciones a lo largo de la última década en coordinación con un importante abanico de programas adicionales. Desde la asunción del OPAC web a finales de los noventa, hemos pasado a un catálogo en línea que actúa como integrador de recursos y que permite, entre otras funcionalidades: añadir contenido enriquecido al registro bibliográfico, acceder a servicios de referencia virtual, actuar como metabuscador, integrar enlaces o permitir su acceso a través de dispositivos móviles.

Las empresas del sector presentan desarrollos e innovaciones constantes con OPAC en los que se trasluce la influencia que tienen las herramientas genéricas de búsqueda en la Web como patrón de adaptación a las preferencias de acceso a la información de los usuarios. Así, aparecen entornos más simples, el empleo de menús o la búsqueda avanzada parecen haber quedado relegados, incluso en los catálogos de bibliotecas universitarias o especializadas, y se 
han incorporado funcionalidades avanzadas, ordenación de resultados por relevancia, navegación por facetas, tecnología fuzzy, búsquedas en lenguaje natural, etcétera.

A los productos comerciales se han de sumar los proyectos open source, con $\mathrm{Koha}^{2}$ a la cabeza. Conviene hacer una mención especial en este punto al proyecto eXtensible Catalog (XC), ${ }^{3}$ alojado en la Universidad de Rochester y financiado por la Fundación Andrew W. Mellon Foundation Scholarly Communications Program, que trabaja en el diseño y desarrollo de un conjunto de aplicaciones de código abierto para bibliotecas. XC software está diseñado como un conjunto de componentes más que como un sistema tradicional monolítico, de modo que la biblioteca instalará la herramienta que se ajuste a su necesidad. El software se dirige a tres áreas: ILS connectivity, metadata management y user interface.

Cabe destacar en España el Grupo de Trabajo del Catálogo Colectivo de Bibliotecas de la Administración General del Estado que ha diseñado el SIGB, Koha-Kobli, ${ }^{4}$ y plantea la creación de un punto de consulta único para las colecciones de las Bibliotecas de la Administración General del Estado y sus organismos públicos. Precisamente, la base para desarrollar el proyecto es eXtensible Catalog, que incluye la mayoría de las funcionalidades requeridas por un catálogo colectivo, la posibilidad de importar metadatos de distintos SIGB a través de la recolección OAI-PMH, la normalización de metadatos de distintas fuentes, la posibilidad de construir interfaces avanzadas de consulta, de descubrimiento y de exposición de datos: web, móvil, linked open data, etc. (Arroyo y Sellès, 2011).

Convenimos con apreciaciones como las de Breeding (2008) para quien el negocio de la automatización de bibliotecas basado en licencias de software propietario se ha resquebrajado, los productos han madurado y el número de implementaciones de SIGB open source ha crecido considerablemente. Sin embargo, las conclusiones del trabajo de Wang (2009) en las que se analizan los elementos clave que afectan a los proyectos de migración de SIGB en bibliotecas universitarias americanas, revelan que a pesar del creciente interés por los sistemas open source más de un $80 \%$ de los encuestados señalaron que no habían considerado la opción de un sistema de código abierto para su centro.

El estudio de JISC y Sconul (2008) habla de un mercado totalmente consolidado, que trata de competir con servicios como Google o Amazon, centrándose en potenciar los valores añadidos que sus SIGB pueden proporcionarles 
a las bibliotecas, respetando los estándares y buscando la interoperabilidad. Los resultados de la encuesta del año 2010 efectuada a unas dos mil bibliotecas de sesenta países (Breeding, 2011), muestran dos productos de código abierto situados entre los mejor posicionados en el ranking de calidad: OPALS y Koha. Las empresas y productos implementados en grandes bibliotecas se situán en un nivel intermedio del ranking, y destaca en este grupo el nivel de satisfacción asignado a Millennium (Innovative Interfaces), Library·Solution (The Library Corporation) y Evergreen (Equinox Software).

Consideramos que, en concreto, los catálogos en línea y las aplicaciones relacionadas con los mismos se verán beneficiados ante una perspectiva más competitiva y abierta al software open source, interfaces no dependientes del sistema y una línea de trabajo centrada en el usuario, dirigida a añadir valor a los datos de la biblioteca, alejándose de las "simples" listas de resultados y detalles de registros bibliográficos para trasladar las colecciones de las bibliotecas híbridas al mundo del usuario. Retomando el estudio de Wang (2009), al que hemos hecho referencia, los resultados subrayan el entusiasmo de los encuestados por extensiones tales como herramientas de descubrimiento que mejoren la experiencia del usuario y funcionalidades más centradas en el usuario, así como tecnologías 2.0 a la hora de valorar los proyectos de migración de sistema de gestión bibliotecario.

Para Saorín (2006, p. 256), los SIGB tradicionales se han visto desbordados por las necesidades tecnológicas de las bibliotecas, y se observa una tendencia a la convergencia entre empresas de software y empresas de contenidos, lo que lo lleva a plantear un modelo de SIGB extendido. En esta línea, Sellés y Serrano-Cobos (2011) son contundentes al señalar que durante décadas, los SIGB se han dedicado a mejorar las necesidades de gestión de una biblioteca (préstamos, compras, catalogación, etc.) pero no a programar funcionalidades propias de un gestor de contenidos (tales como mantener archivos de noticias, gestionar videos, foros, redes sociales, etc.). Además, los SIGB ya no son los únicos protagonistas en la gestión de colección y recursos de la biblioteca, puesto que actualmente las bibliotecas utilizan un amplio abanico de herramientas. Lo que se necesita realmente, apostillan, es una forma de conectar varias familias de software que hacen por separado muy bien lo que tienen que hacer, una forma de hacerlos convivir y de sumar fuerzas (y funcionalidades) en un entorno lo más abierto posible, que asegure una evolución muy dinámica que les permita a las bibliotecas tomar el control de lo que realmente quieren que sea su portal, en función de su política propia, y de la naturaleza y necesidades de sus usuarios.

Estamos de acuerdo con Breeding (2012) al considerar que el modelo tradicional de SIGB no es muy apropiado para la gestión de recursos electrónicos 
y esta circunstancia ha llevado a la proliferación de productos complementarios. Una biblioteca universitaria precisa mantener generalmente un entramado de gestión de gran complejidad en el que conviven diversas plataformas de software y hardware que no siempre interactúan de modo óptimo. Este autor apuesta por un modelo de "plataformas de servicios bibliotecarios" que soportan un conjunto amplio de estándares y modelos de metadatos y se asientan en un conjunto robusto de APIs. OCLC's WorldShare Management Services, Alma de Ex Libris, Serials Solutions Intota, Sierra de Innovative Interfaces o el proyecto open source Kuali OLE pueden ser los llamados a sustituir a los SIGB con los que hoy cuentan todas las bibliotecas académicas.

\section{LA NECESIDAD DE INTEGRAR RECURSOS: LA IMPLEMENTACIÓN DE METABUSCADORES O SISTEMAS DE BÚSQUEDA FEDERADA}

En los últimos años un buen número de bibliotecas han venido implementando metabuscadores o sistemas de búsqueda federada, herramientas diseñadas específicamente para integrar recursos de un modo más eficaz al ofrecido por el catálogo. Esto ha sido viable gracias al asentamiento de los sistemas de enlaces de citas que hacen uso de las ventajas de OpenURL (ANSI/ NISO Z39.88) y a las soluciones tecnológicas aportadas por algunas de las principales empresas del sector que disponen de resolutores de enlaces, aplicaciones específicas para solucionar la compleja red de enlaces establecidos en el tratamiento de recursos electrónicos. SFX de ExLibris, WebBridge LR de Innovative Interfaces, 360 Link de Serials Solutions o Resolver para SirsiDynix ilustran este tipo de herramientas.

Los metabuscadores permiten la búsqueda simultánea y transversal a distintos recursos electrónicos haciendo uso de metadatos, proporcionan la posibilidad de localizar información a través de una única inferfaz, hacen búsquedas sobre diferentes recursos de información (catálogo, bases de datos, colecciones digitales, etcétera.) y también localizan revistas electrónicas y obtienen información, e incluso el texto completo, de sus artículos. Sirven, por tanto, para darle visibilidad a muchos de los recursos de la biblioteca que pasan en gran medida desapercibidos para el usuario.

A finales de la década de los noventa y comienzos del nuevo siglo se consideró la búsqueda federada como solución al acceso a los recursos de la biblioteca, Tennant (2003) se refiere a los metabuscadores como el tipo de herramientas robustas y amigables que necesitan implementar las bibliotecas para competir con la simplicidad y popularidad de Google. 
Algunos de los productos de mayor implantación son MetaLib de la empresa Exlibris, Research Pro de Innovative Interfaces, ZPortal de OCLC, etc. Por su parte, NISO patrocinó la MetaSearch Initiative 5 para tratar de avanzar en soluciones más eficaces para los proveedores de contenidos, para las bibliotecas y para los usuarios de éstas.

El estudio de Belliston, Howland y Roberts (2007) agrupa la bibliografía sobre tecnologías de búsqueda federada en cuatro categorías: debates sobre la conveniencia y/o dificultad de crear una herramienta de búsqueda federada sólida; informes sobre implementaciones concretas de metabuscadores; comparaciones entre productos en el mercado y/o con respecto a Google académico; y propuestas para la implementación de herramientas de búsqueda federada de contenidos específicos. Estos autores subrayan en el conjunto de la literatura revisada la escasez de investigaciones cuantitativas.

Destaca como elemento diferenciador crucial entre catálogos y metabuscadores la posibilidad de estos últimos de realizar búsquedas en el texto completo, superando el carácter restrictivo del catálogo ceñido a la exploración de la referencia bibliográfica. La principal ventaja para el usuario se asienta en la comodidad de emplear una única herramienta para consultar distintos recursos cuya interrogación exigiría manejar diversas interfaces. Los resultados se presentan en una única página que permite además, eliminar duplicados o filtrar y combinar ítems. Las bibliotecas suelen presentar en la misma plataforma los recursos organizados bajo distintas categorías: obras de referencia, recursos a texto completo, categorías temáticas, etcétera.

Con todo, son notables los aspectos problemáticos observados en los metabuscadores. Herrera (2007) se refiere en este punto específico a los criterios de relevancia empleados en la presentación de resultados, así como a las dificultades derivadas del tiempo de respuesta. Játiva (2009) señala la adecuación de estos productos para búsquedas sobre temas muy generales, pero apunta una menor adaptación para efectuar búsquedas de carácter específico. Añade esta misma autora la falta de pertinencia, el ruido que se produce con la recuperación de documentos no relevantes y, muy a menudo, el silencio en la recuperación debido a la abundancia de recursos de información alojados en servidores de proveedores distintos, como circunstancias que conducen a consultas poco efectivas.

El reciente trabajo de Swanson y Green (2011) repasa la literatura que discute la implementación de soluciones de búsqueda federada; parece existir coincidencia en el conjunto de los estudios al señalar que los metabuscadores, tratando de reproducir el patrón de búsqueda impuesto por Google 
partiendo de una caja única, resultan más intuitivos, rápidos y apropiados para el acceso al conjunto de los recursos de la biblioteca, y además, proporcionan visibilidad a los contenidos electrónicos. Sin embargo, las soluciones basadas en búsquedas federadas requieren habilidades y conocimientos que el usuario medio no posee y que lo llevan a tener problemas para interpretar los resultados de una búsqueda dada. A pesar de las ventajas, se constata que los usuarios no son conscientes de las diferencias entre bases de datos, catálogos y otros recursos, una herramienta que en ocasiones es una ayuda indudable, en otras genera dificultades y confusión. Por su parte, Way (2010) subraya la escasez de estudios existentes sobre la influencia de las herramientas de búsqueda federada en el uso de los recursos de la biblioteca.

Para Waren (2007) no está claro que la solución a los consabidos problemas de los catálogos sea la tecnología empleada por los metabuscadores. Enumera, entre otros problemas, el hecho de que un importante porcentaje de recursos de texto completo no puedan ser integrados adecuadamente en el entorno del metabuscador, asimismo, plantea dificultades para establecer series de recursos para diversas subcategorías como las correspondientes al Derecho o las Humanidades. Argumenta además, que el concepto de relevancia de los sistemas federados resulta engañoso, dado que son los primeros resultados devueltos los que aparecen ordenados por relevancia y no el conjunto de todos los resultados obtenidos en una búsqueda, igualmente parecen más eficaces algoritmos de relevancia como los empleados por buscadores académicos en los que se atiende al número de citas recibidas y no sólo la frecuencia de aparición de los términos. En su opinión, la búsqueda federada precisa todavía recorrer un largo camino para ofrecer el esperado acceso transparente a la literatura académica.

Los estudios llevados a cabo sobre metabuscadores parecen coincidir en que, a un resultando sistemas imperfectos, el ahorro de tiempo que supone para los usuarios es percibido por éstos como una utilidad potencial significativa frente a la búsqueda en el catálogo tradicional.

\section{Nueva generación de catálogos. LA APUESTA POR LAS PLATAFORMAS DE DESCUBRIMIENTO}

Tradicionalmente, los OPAC de los SIGB propietarios ofrecían mínimas posibilidades de personalización; sin embargo hoy, como ya hemos apuntado, las bibliotecas pueden adquirir complementos adicionales, ya sean de carácter comercial o de software libre, para añadir mejoras funcionales en el catálogo, sin que podamos hablar por ello de verdaderos o completos catálogos de 
última generación. La aparición de estos productos comienza a mediados de la década del 2000. Ex Libris lanzó Primo, Innovative Interfaces presentó Encore, VTLS introdujo Visualizer, etcétera.

Lo realmente novedoso en este punto es que a los nuevos módulos de OPAC que persiguen la inclusión de registros enriquecidos, y especialmente elementos 2.0 de participación social, se unieron muy pronto soluciones con herramientas específicas que conforman una nueva interfaz desvinculada del OPAC; se trata de aplicaciones que actúan como envoltorio o máscara del catálogo, remplazando al módulo de OPAC.

Atendiendo a Yang y Hofmann (2011) la expresión "next generation catalog" se acuñó a mediados de la primera década de este siglo XXI. Si bien el término fue empleado con anterioridad de modo impreciso, es el trabajo de Antelman, Lynema y Pace de 2006 el que cristaliza la expresión, y será un año más tarde cuando Breeding (2007) describa las características de esta nueva generación de catálogos.

La literatura les adscribe a estos sistemas los elementos distintivos siguientes:

- Punto de entrada único a los recursos de la biblioteca. El catálogo debería consistir en una caja de búsqueda única o búsqueda federada a todos los materiales de la biblioteca.

- Interfaz web de diseño actual, semejante a las disponibles en webs comerciales.

- Contenido enriquecido. Deben incluir imágenes de las cubiertas, enlaces a tablas de contenidos, a sumarios, a descripciones, a valoraciones y a nubes de etiquetas, así como a contenidos elaborados por la propia biblioteca o por fuentes comerciales.

- Navegación por facetas que favorece la acotación de la búsqueda. Catálogos capaces de desplegar los resultados de la búsqueda en grupos de categorías como términos de materia, fechas, idiomas, disponibilidad del ítem, tipos de formatos, localización, etcétera.

- Caja de búsqueda simple por palabra clave con enlace a la búsqueda avanzada en cada una de las páginas que recorre el usuario a medida que navega o efectúa búsquedas.

- Resultados ordenados por relevancia. Los criterios de relevancia empleados en los catálogos han sido objeto de numerosas críticas. Los nuevos catálogos deberían conseguir una mayor precisión, para ello podrán tener en cuenta la frecuencia de préstamo o la existencia de múltiples copias.

- Mecanismos de autocorrección, comprobaciones ortográficas y alternativas de corrección utilizando expresiones del tipo: “¿quiso decir?”. 
- Recomendaciones y/o materiales relacionados, aprovechando la información de los ficheros log, los catálogos deberían recomendar libros a los lectores mediante enlaces a lecturas recomendadas o locuciones como: "los usuarios que tomaron en préstamo este libro también se llevaron en préstamo los siguientes...”.

- Contribuciones del usuario. Los nuevos catálogos les permiten a los usuarios añadir datos a los registros bibliográficos: descripciones, resúmenes, reseñas, críticas, comentarios, etiquetas, puntuaciones, etcétera.

- Fuentes RSS, deberían ofrecerle al usuario la posibilidad de suscribirse a canales RSS para estar actualizado sobre nuevos libros adquiridos, listas de libros más prestados, etcétera.

- Integración con redes sociales, los catálogos permiten compartir enlaces de los ítems de la biblioteca con sitios 2.0.

- Enlaces persistentes, cada registro contará con un identificador o localizador estable.

Játiva (2009) se refiere a OPAC de última generación concebidos como plataformas de integración de las distintas fuentes y los variados servicios de información de la biblioteca. La clave de esta generación de catálogos, según esta autora, es la ampliación y el desarrollo del contenido; es decir, los recursos y servicios, así como la transformación del continente, esto es, la interfaz que pasa a convertirse en un portal integrador de fuentes y un espacio de comunicación, creación e intercambio de información para el usuario.

Como subrayan Yang y Warner (2010) observamos distintos términos para hacer referencia a estas herramientas, sirve de ejemplo OPAC autónomo, capa de descubrimiento, interfaz de capa de descubrimiento, OPAC de sustitución o última generación de catálogos. Gross y Sheridan (2011), siguiendo la propuesta de Breeding (2010b) se inclinan por la denominación de interfaces de descubrimiento (discovery interfaces). Coincidimos en la preferencia por este término dado que, como señala el propio Breeding, el objetivo de estas plataformas es facilitar el acceso al conjunto de las colecciones de la biblioteca, no sólo a los contenidos incluidos en el catálogo de la biblioteca, tradicionalmente contenidos gestionados por el sistema integrado de bibliotecas. Se trata de ayudar al usuario a descubrir el contenido de la biblioteca en todos los formatos, independientemente de si residen dentro de la biblioteca física o entre sus colecciones digitales.

Atendiendo a Vaughan (2011), OCLC WorldCat Local, ${ }^{6}$ lanzado en noviembre de 2007, puede considerarse el primer servicio de descubrimiento de 
escala web. En julio de 2009 la empresa Serials Solutions anunció el desarrollo de Summonm, ${ }^{7}$ como herramienta de descubrimiento (web scale discovery service). Pronto hicieron su aparición productos semejantes desarrollados por otras empresas del sector: Ebsco's Discovery Service de Ebsco ${ }^{8}$ en enero de 2010, Encore Synergy de Innovative Interfaces unos meses más tarde o Primo Central Index ${ }^{9}$ de ExLibris lanzado en junio de 2010.

En las bibliotecas académicas españolas resultó pionera la introducción de AquaBrowser ${ }^{10}$ en los centros dependientes de la Fundación Universitaria San Pablo CEU, abandonando su OPAC en favor de uno de versión autónoma fundamentado en mapas conceptuales.

Entre las herramientas de código abierto, resulta destacable el lanzamiento de la interfaz de descubrimiento de eXtensible Catalog Organization -XC Drupal Toolkit- ${ }^{11}$ entre cuyas funcionalidades destaca la personalización de la herramienta en su conjunto así como de las facetas de navegación, la conexión directa con el módulo de circulación del SIGB o el estar sustentada en los modelos FRBR / RDA.

Las interfaces de descubrimiento, a diferencia de los metabuscadores, pre-recolectan los contenidos en un índice único que elimina la necesidad de combinar resultados, permite mayor rapidez de respuesta, una más fácil eliminación de duplicados y un mejor ranking de relevancia. Con todo, en opinión de algunos autores como Yang y Wagner (2010), la distinción efectiva entre herramientas de búsqueda federada y plataformas de descubrimiento es cada vez más imprecisa.

Las empresas que comercializan plataformas de descubrimiento han negociado con los proveedores de contenidos para permitirles preindizar los metadatos de los ítems o los contenidos a texto completo. Se trata de sistemas capaces de indizar un enorme abanico de contenidos, ya estén alojados en el servidor local o en servidores remotos, registros incluidos en el SIGB, en el repositorio institucional, en colecciones digitales, en contenidos de bases de datos de la propia institución o contratadas bajo licencia, en ítems a texto completo procedentes de distribuidores de libros y revistas electrónicas.

Las herramientas de descubrimiento presentan por defecto una caja de búsqueda simple e intuitiva que puede complementarse con utilidades de búsqueda avanzada si el usuario así lo requiere. Las interfaces incluyen navegación por facetas, ordenación personalizada de resultados por los campos

7 http://www.serialsolutions.com/discovery/summon/

8 http://ebscohost.com/discovery

9 http://www.exlibrisgroup.com/category/PrimoCentral

10 http://www.serialsolutions.com/discovery/aquabrowser/

11 http://www.extensiblecatalog.org/software/drupaltoolkit/demo 
como fecha de publicación, tipo de ítem, texto completo, etc. Además, ofrecen a las bibliotecas posibilidades notables de personalización y extensión de la herramienta con respecto a la arquitectura cerrada de los SIGB.

A pesar de su corta existencia, contamos ya con diversos estudios que analizan estas plataformas. Aunque con límites imprecisos, se pueden agrupar estos trabajos en dos grandes bloques, de un lado las experiencias de implementación y análisis de funcionalidades, y, del otro los estudios de usabilidad.

Dentro del primer bloque, el informe de Vaughan (2011) describe el modelo de investigación, evaluación y propuesta utilizado en la University of Nevada, Las Vegas, valiéndose de un Grupo de trabajo específico para seleccionar una herramienta adecuada a las características de la institución. Considera que las plataformas de descubrimiento representan una evolución y, quizás, una revolución para el usuario final y para las propias colecciones bibliotecarias. Powers, Briceño-Rosales, Fernandez y Varnum (2011) argumentan la elección de las herramientas EDS de EBSO, WorldCat de OCLC, Primo de Ex Libris y Summon de Serials Solutions en sus respectivas universidades y los cambios percibidos por los usuarios derivados de su implementación.

Yang y Wagner (2010) analizan plataformas de descubrimiento atendiendo a la adopción o no de doce funcionalidades distintivas de esa nueva generación de catálogos. Las conclusiones señalan taxativamente que los 17 productos objeto de evaluación no pueden ser considerados realmente como nueva generación de catálogos, dado que ninguno de ellos implementa esas doce características requeridas; en concreto las opciones de búsqueda federada y relevancia basada en la transacción de datos no se hallaban implementadas en los programas estudiados. Asimismo cabe destacar que las tres plataformas mejor posicionadas en el estudio se corresponden con herramientas de código abierto, en concreto: LibraryFind, Vufind y Scriblio.

El informe de OCLC, Prescott y Erway (2011), señala la necesidad de mejorar las plataformas de descubrimiento incorporando herramientas de navegación y filtrado. Para esto, los datos de los campos de autoridades existentes, autores, lugares, temas y tipos de materiales son los candidatos perfectos para ayudar a los usuarios a saltar en el sistema evitando la intimidación que puede producir la caja de búsqueda vacía. Estos términos controlados pueden implementarse para actuar como guías sensibles al contexto que ayuden al usuario a seleccionar y navegar a través de los resultados de búsqueda.

Por su parte, en el bloque de estudios de usabilidad situamos el trabajo de Way (2010), en el que se explora el impacto de Summon en el uso de los recursos de la biblioteca tras un semestre de implementación. Los resultados del análisis sugieren que la herramienta tuvo un impacto considerable en el 
comportamiento de los usuarios y en el uso de las colecciones de la biblioteca durante ese periodo. A pesar de las limitaciones, el estudio muestra que los usuarios fueron capaces de identificar y seleccionar contenidos de libros, al aumentar las estadísticas de acceso al catálogo; se aprecia un incremento considerable en el uso de los recursos a texto completo de la biblioteca, no obstante, el uso de bases de datos bibliográficas continúa mostrando una línea descendente.

Emanuel (2011) realiza un análisis de usabilidad de VuFind y concluye que se trata de una herramienta cuya interfaz valoran muy positivamente los usuarios a pesar de haber observado ciertas limitaciones, entre las que destacan las carencias detectadas en la búsqueda multimedia. Por su parte Ramdeen y Hemminger (2011) analizan la experiencia del usuario en la búsqueda de información al confrontar un catálogo de interfaz tradicional y un catálogo con funcionalidades de búsqueda facetada -Endeca-. Su conclusión es que en su totalidad, los participantes en el estudio mostraban una clara preferencia por la interfaz de búsqueda con facetas, por la facilidad de su uso y porque las facetas dinámicas refuerzan la confianza en la selección de la respuesta correcta. Empleando las facetas los usuarios pueden ampliar o restringir sus búsquedas manteniendo el contexto de la búsqueda efectuada inicialmente.

Gross y Sheridan (2011) analizan en su estudio el comportamiento y la percepción de un conjunto de estudiantes universitarios en el empleo de Summon. Los resultados subrayan la seguridad de los usuarios al interaccionar con una interfaz amigable, sin embargo hacen hincapié en que esto no implica que los estudiantes comprendan adecuadamente el proceso de búsqueda o la necesidad de evaluar los recursos de información. Por su parte Kenney (2011), relata la experiencia de los usuarios de la Biblioteca de Arte y Humanidades de la Universidad de Liverpool en el empleo de EDS de Ebsco. Se señala que la interfaz de descubrimiento les permite a los investigadores experimentados localizar contenidos que de otro modo hubiesen pasado desapercibidos, con lo cual muestran una gran ventaja en áreas académicas donde se valora la investigación interdisciplinar.

Los estudios llevados a cabo hasta el momento, sin soslayar los aspectos mejorables de estas herramientas, parecen confirmar el salto cualitativo que suponen las plataformas de descubrimiento para el usuario final. Sin duda, se manifiesta imprescindible contar con estudios cuantitativos que complementen los trabajos de usabilidad.

El exhaustivo análisis de Fry y Rich (2011) en la Bowling Green State University de Ohio concluye que resulta crucial para los estudiantes contar con una única caja de búsqueda para acceder a los contenidos de la biblioteca 
y apuesta por las plataformas de descubrimiento como la mejor opción para la mayor parte de los usuarios. Estas interfaces evitan las dudas a la hora de seleccionar entre subconjuntos de información (catálogo versus bases de datos) o el formato buscado (artículo versus título de revista). En su opinión, resultará fundamental para el éxito de estas herramientas el desarrollo de adecuados rankings (rangos) de relevancia, la presentación de los resultados facetados y la capacidad de búsqueda en profundidad en los contenidos de las bases de datos.

\section{Plataformas de descubrimiento en las BIBLIOTECAS UNIVERSITARIAS ESPAÑOLAS.}

Son 71 bibliotecas universitarias, de carácter tanto público como privado, las que conforman Rebiun ${ }^{12}$ (Red de Bibliotecas Universitarias Españolas). Se ha procedido a revisar la sede web de cada una de estas instituciones para conocer el estado actual de implementación de interfaces de descubrimiento en el ámbito académico español. La distribución queda reflejada en la figura siguiente.
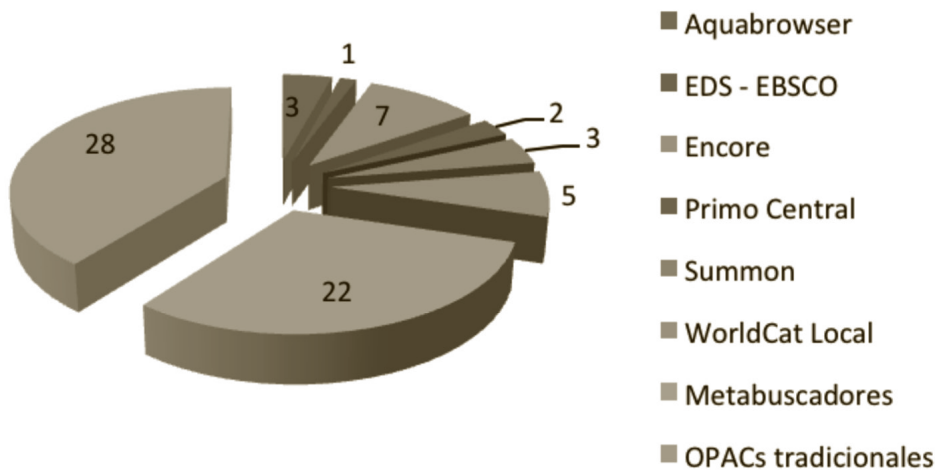

Fig. 1. Distribución de herramientas de búsqueda

Podemos calificar de incipiente la incorporación de interfaces de descubrimiento en las universidades españolas, si bien consideramos destacable tanto el número de bibliotecas que presentan soluciones de búsqueda más allá de los catálogos asociados al SIGB empleado, como la tradición académica de las mismas. 
Encore de Innovative Interfaces parece la solución preferida de modo muy particular por un buen número de bibliotecas universitarias andaluzas. Se ha constatado en el momento del análisis la implantación de Summon en las universidades Complutense de Madrid, La Rioja e Internacional de Andalucía. Aquabrowser es la plataforma implementada en los tres centros de la Fundación Universitaria San Pablo CEU, y EDS-Ebsco es la interfaz instalada en la biblioteca de la Universidad de Navarra.

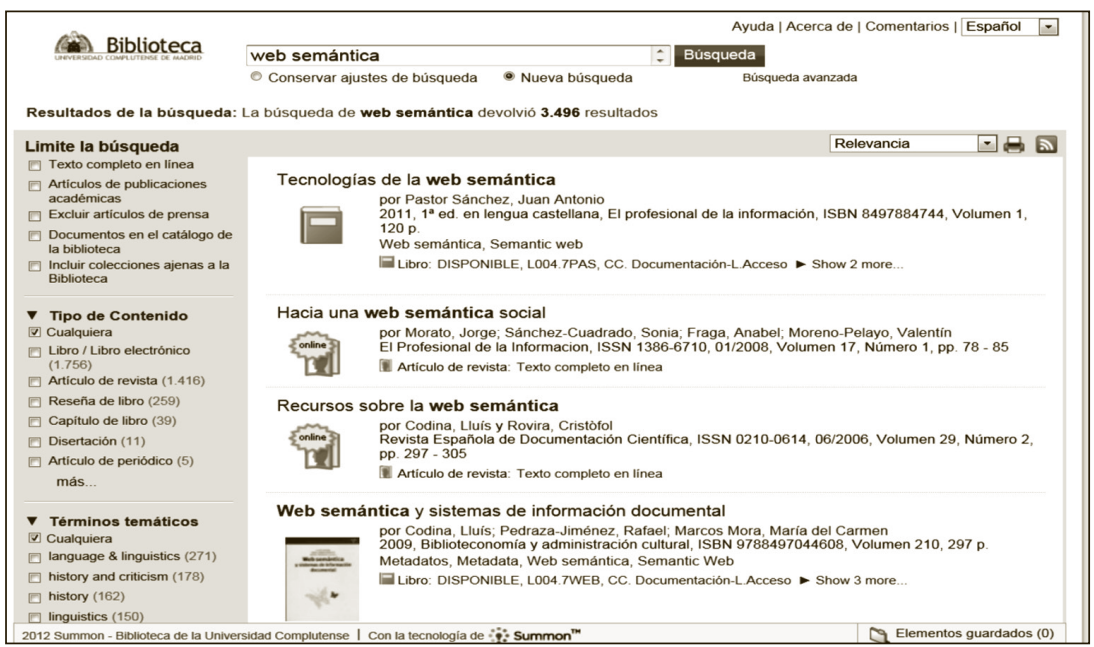

Fig. 2. Implementación de Summon en la Universidad Complutense de Madrid

Cabe mencionar la decisión del Consorcio BUCLE, integrado por las Universidades de Burgos, León, Salamanca y Valladolid, dirigida a generar su catálogo colectivo a partir de WorldCat Local, esta misma plataforma de OCLC ha sido la elegida por la Universidad de Alicante. 


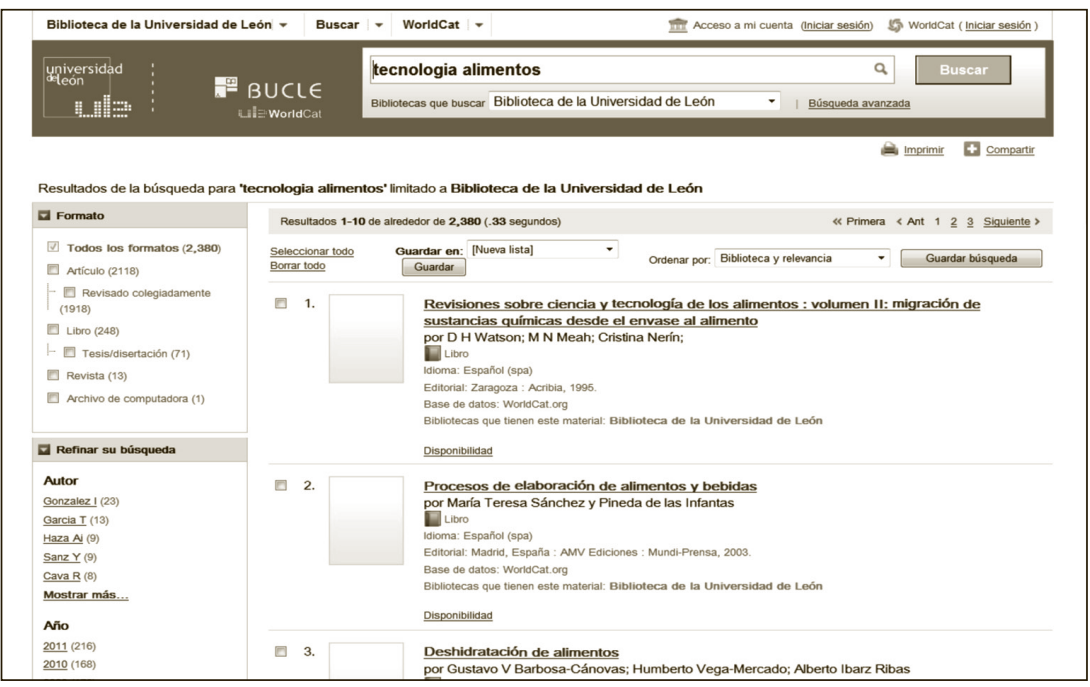

Fig. 3. WorldCat Local en la Universidad de León

En el caso de Primo Central se ha verificado su instalación en la Universidad Politécnica de Valencia y en los centros del IE, además, creemos oportuno puntualizar, dado que MetaLib es el metabuscador incorporado mayoritariamente en las bibliotecas universitarias españolas, y atendiendo a la política de la empresa, que tal vez incorporen esta plataforma varios centros más. MetaLib+ es el servicio Primo para los usuarios de MetaLib. Ya existe una implementación de Metalib+ en España, la del Consorci de Biblioteques Universitàries de Catalunya (CBUC), BuscaRE, ${ }^{13}$ que afecta, por tanto, al conjunto de las universidades públicas catalanas y a la Oberta de Catalunya. Se han incluido, además, varias colecciones en castellano en Primo Central: SCIELO, RACO, RECERCAT y TDX.

Creemos que a pesar del carácter transitorio de los datos mostrados, esta aproximación realizada nos permite confirmar el esfuerzo de las instituciones académicas por ofrecer una herramienta de búsqueda única para la multitud de recursos que conforma una colección académica en la actualidad. La articulación definitiva del modelo puede depender en muchos casos de las políticas comerciales de las empresas que ya cuentan con herramientas de búsqueda previamente implementadas en las instituciones. No se ha observado ninguna instalación con interfaces de descubrimiento de software libre.

13 http://primo-service.hosted.exlibrisgroup.com/primo_library/libweb/action/search.do?vid =CBUC_V1 


\section{Consideraciones finales}

Las bibliotecas universitarias ofrecen un conjunto fragmentado de sistemas para buscar información y a pesar de los desarrollos tecnológicos dirigidos a la integración de recursos y del denodado trabajo de la comunidad bibliotecaria para darle visibilidad a las colecciones, resulta recurrente la consideración poco favorable de los usuarios hacia los OPAC. Los metabuscadores han demostrado ser herramientas potentes y útiles pero, por su propia naturaleza no logran conformar esa caja de búsqueda única que demanda la comunidad académica para localizar información en el conjunto de las colecciones ofertadas.

El planteamiento observable en la actualidad parece dirigirse hacia la implementación de interfaces de descubrimiento que permitirían "abandonar" el OPAC convencional en favor de una plataforma autónoma que facilite el acceso al conjunto de los materiales de la biblioteca emulando el comportamiento de un buscador web. Los estudios sobre estas herramientas efectuados hasta el momento, aun señalando sus puntos débiles, son notablemente positivos en la valoración de éstos y en la experiencia de búsqueda que le proporcionan al usuario.

La situación en las bibliotecas universitarias españolas manifiesta una apuesta decidida por herramientas de búsqueda complementarias al catálogo, y en esta línea se entiende la implementación en los últimos meses de interfaces de descubrimiento en una proporción que podemos calificar de significativa.

Consideramos prioritario estudiar los desarrollos de estas plataformas, su adaptación al comportamiento real de los usuarios, y su influencia en el uso y visibilidad de las colecciones disponibles en la biblioteca.

\section{Bibliografía}

Antelman, K.; Lynema, E.; Pace, A. K. (2006), Toward a Twenty-first Century Library Catalog, en Information Technology \& Libraries, vol. 25, núm. 3, pp. 128-139, disponible en: http://eprints.rclis. org/bitstream/10760/8177/1/antelman_lynema_pace.pdf

Arriola Navarrete, O.; Tecuati Quechol, G.; González Herrera, G. (2011), "Software propietario vs software libre: una evaluación de sistemas integrales para la automatización de bibliotecas", en Investigación bibliotecológica, vol. 25, núm. 54, pp. 37-70, disponible en: http://www.ojs.unam.mx/index.php/ibi/article/download/27 $480 / 25468$ 
Arroyo Fernández, D.; Sellés, A. (Coords.) (2011), Punto de consulta único de las Bibliotecas de la Administración General del Estado: informe del proyecto. Madrid: Subdirección General de Coordinación Bibliotecaria, disponible en: http://hdl.handle.net/10421/ 5041

Belliston, C. J.; Howland J. L.; Roberts, B. C. (2007), "Undergraduate Use of Federated Searching: A Survey of Preferences and Perceptions of Value-added Functionality", en College and Research Libraries, vol. 68, pp. 472-486.

Bissels, G. (2008), "Implementation of an open source library management system: Experiences with Koha 3.0 at the Royal London Homoeopathic Hospital", en Program: Electronic Library and Information Systems, vol. 42, núm. 3, pp. 303-314.

Borgman, C. L. (1986), "Why are online catalogs hard to use? Lessons learned from information retrieval studies", en Journal of the American Society for Information Science, vol. 37, núm. 6, pp. 387 400.

(1996), "Why are online catalogs still hard to use?", en Journal of the American Society for Information Science, vol. 47, núm. 7, pp. 493-503.

Breeding, M. (2007), "Next-Generation Library Catalogs", en Library Technology Reports, vol. 43, núm. 4, disponible en: http://alatechsource.metapress.com/content/1192704qg105/?p=8e05b384f5eb 4256a6b1314afcdc2b33\&pi=36

(2008), "Automation System marketplace 2008: opportunity out of turmoil”, en Library Journal, vol. 133, núm. 1, disponible en: http://www.libraryjournal.com/article/CA6542440.ht$\mathrm{ml}$ lishing, 2010. (2010a), Next-Gen Library Catalogs, London: Facet Pub, (2010b), "State of the Art in Library Discovery 2010" en Computers in Libraries, vol. 30, núm. 1, pp. 31-35, disponible en: http://www.librarytechnology.org/ltg-displaytext.pl?RC=14574 , (2011), "Perceptions 2010: An International Survey of Library Automation”, en Library Technology Guides, disponible en: http://www.librarytechnology.org/perceptions2010.pl , (2012), Current and future trends in information technologies for information units, El profesional de la Información, vol. 21, núm. 1, pp. 9-15.

Byrum, J. D.; Williamson, D. W. (2006), "Enriching traditional cataloging for improved access to information: Library of congress tables of contents projects", en Information technology and libraries, vol. 25, núm.1, pp. 4-11.

Calhoun, K. (2006), The Changing Nature of the Catalog and its Integration with Other Discovery Tools. Final Report, Prepared for the Library of Congress, disponible en: http://www.loc.gov/catdir/ calhoun-report-final.pdf 
Calhoun, K. et al (2009), Online Catalogs: What Users and Librarians Want. An OCLC Report, disponible en: http://www.oclc.org/reports/onlinecatalogs/fullreport.pdf

Campbell, D. G.; Fast, K. V. (2004), "Panizzi, Lubetzky, and Google: How the Modern Web Environment is Reinventing the Theory of Cataloguing", en Canadian Journal of Information E Library Sciences, vol. 28, núm. 3, pp. 25-38, disponible en: http://www.caisacsi.ca/proceedings/2004/campbell_2004.pdf

CIBER, "Centre for Information Behaviour and the Evaluation of Research. (2008)", en Information Behaviour of the researcher of the future, School of Library, Archive and Information Studies, University College London, disponible en: http://www.jisc.ac.uk/me$\mathrm{dia} /$ documents/programmes/reppres/gg_final_keynote_11012008.pdf

De Rosa, C. et al (2006), College Students' Perceptions of Libraries and Information Resources: A Report to the OCLC Membership, Dublin, Ohio: OCLC, disponible en: http://www.oclc.org/reports/pdfs/ studentperceptions.pdf

El-Sherbini, M. ; Wilson, A. J. (2007), "New Strategies for Delivering Library Resources to Users: Rethinking the Mechanisms in which Libraries are Processing and Delivering Bibliographic Records", en The Journal of Academic Librarianship, vol. 33, núm. 2, pp. 228-242.

Emanuel, J. (2011), "Usability of the VuF ind Next-Generation Online Catalog", en Information Technology and Libraries (ITAL), vol. 30, núm. 1, disponible en: http://www.ala.org/lita/ital/sites/ala. org.lita.ital/files/content/30/1/pdf/emanuel.pdf

Fast, K. V. ; Campbell, D. G. (2004), "I still like Google: University Student Perceptions of Searching OPACs and the Web", en Proceedings of the ASIS Annual Meeting 2004, Medford, NJ: Information Today, vol. 41, pp. 138-146.

Flynn, M. (2010), "From Dominance to Decline? The Future of Bibliographic Discovery, Access and Delivery", en World Library and Information Congress: 76th IFLA General Conference and Assembly 10-15 August 2010, Gothenburg, Sweden, disponible en: http:// www.ifla.org/files/hq/papers/ifla76/71-flynn-en.pdf

Fry, A. ; Rich, L. (2011), "Usability Testing for e-Resource Discovery: How Students Find and Choose e-Resources Using Library Web Sites" en The Journal of Academic Librarianship, vol. 37, núm. 5, pp. 386-401.

Garza, A. (2009), "Next generation OPACs: part 2. From OPAC to CMS: Drupal as an extensible library platform" en Library Hi Tech, vol. 27, núm. 2, pp. 252-267.

Gross, J. ; Sheridan, L. (2011), "Web scale discovery: the user experience”, en New Library World, vol. 112, núm. 5/6, pp. 236- 247.

Herrera, G. (2007), "Metasearching and beyond: implementation experiences and advice from an Academic Library", en Information Technology and libraries, vol. 26, núm. 2, pp. 44-52. 
Játiva Miralles, M. V. (2009), "El catálogo: un recurso en expansión”, en Anales de Documentación, vol. 12, pp. 69-92, disponible en: http://revistas.um.es/analesdoc/article/view/70251

Jisc (2010), Improving the resource discovery experience. Executive overview, disponible en: http://www.jisc.ac.uk/media/documents /publications/programme/2010/improvingresourcediscoveryexperience.pdf

Jisc \& Sconul. (2008), Library Management Systems Study. An Evaluation and horizon scan of the current library management systems and related systems landscape for UK bigher education. Study Report, disponible en: http://www.jisc.ac.uk/media/documents/ programmes/resourcediscovery/lmsstudy.pdf

Kenney, B. (2011), "Liverpool's Discovery: A university library applies a new search tool to improve the user experience", en $\mathrm{Li}$ brary Journal, February, disponible en: http://www.libraryjournal.com/lj/home/888965-264/liverpools_discovery.html.csp

Mann, T. (2006), The Changing Nature of the Catalog and Its Integration with Other Discovery Tools. Final Report. March 17, 2006. Prepared for the Library of Congress by Karen Calhoun, A Critical Review. April, disponible en: http://guild2910.org/AFSCMECalhoun ReviewREV.pdf

Marcum, D. B. (2006), The Future of Cataloging, en Library Resources E Technical Services, vol. 50, núm. 1, pp. 5-9.

Markey, K. (2007a), “The Online Library Catalog: Paradise Lost and Paradise Regained?”, en D-Lib Magazine, vol. 3, núm. 1-2, disponible en: http://www.dlib.org/dlib/january07/markey/01mar key.html

(2007b), "Twenty-five years of end-user searching, Part 1: Research findings", en Journal of the American Society for Information Science and Technology, vol. 58, núm. 8, pp. 1071-1081. , (2007c), "Twenty-five years of end-user searching, Part 2: Future research directions", en Journal of the American Society for Information Science and Technology, vol. 58, núm. 8, pp. 1123. 1130.

Novotny, E. (2004), “I Don't Think I Click: A Protocol Analysis Study of Use of a Library Online Catalog in the Internet Age", en College \& Research Libraries, vol. 65, núm. 6, pp. 525-537. Disponible en: http://crl.acrl.org/content/65/6/525.full.pdf+html

Olson, T. A. (2007), "Utility of a faceted catalog for scholarly research", en Library Hi Tech, vol. 25, núm. 4, pp. 550-561.

Pace, A. K. (2004), "Dismantling Integrated Library Systems", en Library Journal, vol. 19, núm. 2, pp. 34-36.

Powell, C. K. (2008), "OPAC integration in the era of mass digitization: the MBooks experience”, en Library Hi Tech, vol. 26, núm. 1, pp. 24-32. 
Powers, A. C.; Briceño-Rosales, Z. C.; Fernandez, R.; Varnum, K. (2011), "Discovering What Works: Librarians Compare Discovery Interface Experiences”, en Library Journal Reviews, December, disponible en: http://reviews.libraryjournal.com/2011/12/ reference/discovering-what-works-librarians-compare-discoveryinterface-experiences/

Prescott, L.; Erway, R. (2011), Single search: the quest for the Holy Grail. Dublin, Ohio: OCLC Research, disponible en: http://www. oclc.org/research/publications/library/2011/2011-17.pdf

Ramdeen, S.; Hemminger, B. M. (2011), "A Tale of Two Interfaces: How Facets Affect the Library Catalog Search Experience”, en Journal of the American Society for Information Science and Technology (Published online Nov. 28th 2011).

Research Information Network (2009), Creating Catalogues: Bibliographic Records in a Networked World. A Research Information Network report, disponible en: http://www.rin.ac.uk/creatingcatalogues

Rodríguez Bravo, B.; Alvite Díez, M. L. (2004), "Propuesta metodológica de evaluación de interfaces de OPACs. INNOPAC versus UNICORN", en Revista Española de Documentación Científica, vol. 27, núm. 1, pp. 30-44.

Rowley, J. E. (1998), The electronic library, 4th ed. London: Library Association.

Salse Rovira, M. (2005), "Panorámica dels sistemas de gestió de biblioteques al segle XXI", en BiD: textos universitaris de Biblioteconomia i Documentació, núm. 15, disponible en: http://www2.ub. edu/bid/consulta_articulos.php?fichero $=15$ salse.htm

Saorín Pérez, T. (2006), Modelo conceptual para la automatización de bibliotecas en el contexto digital, Tesis doctoral dirigida por José Vicente Rodríguez Muñoz, Universidad de Murcia, disponible en: http://tdx.cat/bitstream/handle/10803/10903/SaorinPerez. pdf? sequence $=1$

Sauperl, A. ; Saye, J. D. (2009), "Have we made any progress? Catalogues of the future revisited", en Journal of Documentation, vol. 65, núm. 3, pp. 500-514.

Sellés-Carot, A. ; Serrano-Cobos, J. (2011), "Del opac extendido a la biblioteca expandida: una realidad al fin", en Notas ThinkEPI, disponible en: http://www.thinkepi.net/del-opac-extendido-a-la-biblioteca-expandida-una-realidad-al-fin

Swanson, T. A.; Green, J. (2011), "Why We Are Not Google: Lessons from a Library Web site Usability Study", en The Journal of Academic Librarianship, vol. 37, núm.3, pp. 222-229.

Tennant, R. (2003), “The Right Solution: Federated Search Tools”, en Library Journal, vol. 128, núm. 11, pp. 28-30.

, (2005a), "Digital libraries: Google, the Naked Emperor", en Library Journal, August, disponible en: http://www.libraryjournal.com/article/CA633310.html 
(2005b), Digital Libraries: "Lipstick on a Pig", en Library Journal, April, disponible en: http://www.libraryjournal.com/article/CA516027.html

University of California Libraries (2005), Rethinking How We Provide Bibliographic Services for the University of California: Final Report, December 2005, Bibliographic Services Task Force, disponible en: http://libraries.universityofcalifornia.edu/sopag/BSTF/Final.pdf

Vaughan, J. (2011), Investigations into Library Web Scale Discovery Services. Information Technology and Libraries Preprint, disponible en: http://www.ala.org/lita/ital/sites/ala.org.lita.ital/files/content/prepub/vaughan2011.pdf

Way, D. (2010), “The Impact of Web-scale Discovery on the Use of a Library Collection" en Serials Review, vol. 36, núm. 4, pp. 214 220.

Wang, Z. (2009), “Integrated Library System (ILS) Challenges and Opportunities: A Survey of U.S. Academic Libraries with Migration Projects", en The Journal of Academic Librarianship, vol. 35, núm. 3, pp. 207-220.

Waren, D. (2007), Lost in Translation: The Reality of Federated Searching, en Australian Academic E Research Libraries, vol. 38, núm. 4, pp. 258-269, disponible en: http://alia.org.au/publishing/ aarl/38/ARRL.Vol38.No4.2007.pdf

Wells, D. (2007), "What is a library OPAC?”, en The Electronic Library, vol. 25, núm. 4, pp. 386-394.

Yang, S. Q. ; Hofmann, M. A. (2011). Next generation or current generation?: A study of the OPACs of 260 academic libraries in the USA and Canada. en Library Hi Tech, vol. 29, n. 2, p. 266-300.

Yang, S. Q. ; Wagner, K. (2010), "Evaluating and comparing discovery tools: how close are we towards next generation catalog?", en Library Hi Tech, vol. 28, núm. 4, pp.690- 709.

Zumer, M. (2007), "Amazon: competition or complement to OPACs", en Bid: textos universitaris de Biblioteconomia $i$ Documentació, núm. 19, disponible en: http://www2.ub.edu/bid/consulta_articulos.php?fichero=19zumer $2 \cdot$ htm 